\title{
Biomineralization at Interfaces Revealed with 4D Electron and Atom Probe Tomographies.
}

\author{
Xiaoyue Wang ${ }^{1}$, Brian Langelier ${ }^{1}$, Anders Palmquist ${ }^{2}$ and Kathryn Grandfield ${ }^{1}$ \\ 1. Department of Materials Science and Engineering, McMaster University, Hamilton, Canada. \\ 2. Department of Biomaterials, Sahlgrenska Academy, University of Gothenburg, Gothenburg, Sweden.
}

Biomineralization at engineered interfaces has huge implications not only for osseointegration of bone and dental implants in health sciences but also for biomimetic synthetic materials, which have wide applications in the energy and environmental sectors. Resolving the spatial and chemical structure of the interface of hierarchical biominerals, such as human bone interfacing to titanium, on the sub-nanometer scale has the potential to shed light on the mechanisms of biomineralization and structure-property relationships. Previous work has unveiled the structure of the bone-implant interface with single-tilt axis electron tomography, however the chemical structure remains unresolved until now [1].

In this work, on-axis rotation tomography, enabling a complete $360^{\circ}$ sample rotation, was used to circumvent the "missing wedge" which is a main source of artifacts in conventional electron tomography [2]. Based on the needle-like sample geometry, correlative 4D electron energy loss spectroscopy (EELS) tomography from the same sample was acquired to offer a further understanding of nanoscale chemical structure at implant interfaces in three dimensions. Atom probe tomography (APT), combing sub-nanometer resolution with chemical sensitivity across the entire periodic table, supports and complements these electron tomographies to strengthen the whole 4D view (spatial plus chemical) of the human bone-implant interface.

A nano-structured titanium implant and surrounding bone was retrieved from the human maxilla after 4 years in service [3]. Using focused ion beam (FIB), specimens from the bone-titanium interface were sharpened into approximately $200 \mathrm{~nm}$ diameter needle-shaped specimens for further investigation. In the scanning transmission electron microscope (STEM) (Titan 80-300, operated at 300kV), sharpened specimens were rotated through $\pm 90^{\circ}$ using an on-axis tomography holder and images were recorded every $2^{\circ}$ on a high-angle annular dark-field (HAADF) detector which provides compositional contrast. After on-axis electron tomography, the needle-shaped sample was put back into the FIB and milled to 50 $\mathrm{nm}$ in diameter. Then, a series of STEM EELS spectrum images and correlative dark-field images were acquired between $\pm 70^{\circ}$ on a double-aberration corrected Titan 80-300 STEM. Chemical maps were extracted for elements $\mathrm{Ca}, \mathrm{C}$, Ti and $\mathrm{O}$, consistent with the mineral and collagen phases of bone, titanium implant, and surface oxide, respectively and reconstructed by applying the alignment and reconstruction algorithm for images to each elemental map. Using visualization software, all elemental maps are merged to identify the organic and inorganic motifs at the interface. Taking advantage of the chemical sensitivity (down to $1 \mathrm{ppm}$ ) of laser-pulsed APT, trace elements and co-localization of ions within specific regions in bone structure and at the material surface were identified.

We present for the first time 4D (spatial + chemical) tomographies of human bone interfacing to nanostructured titanium devices with correlative on-axis electron tomography, chemically sensitive EELS tomography and trace-element sensitive APT. These correlative advanced four-dimensional microscopies provide a foundation for understanding the structure and chemical nature of the interface of hierarchical biominerals, and could be extended to the study of biomineralization mechanism at other 
biological interfaces.

References:

[1] K. Grandfield, S. Gustafsson and A. Palmquist. Nanoscale, 5 (2013), p. 4032.

[2] P.A. Midgley and M. Weyland. Ultramicroscopy, 96 (2003), p. 413.

[3] F.A. Shah et al. Nanomedicine-Nanotechnology Biology and Medicine, 10 (2014), p. 1729.

[4] The authors acknowledge funding from the Natural Sciences and Engineering Research Council of Canada (NSERC) Discovery Grant program and BIOMATCELL VINN Excellence Center of Biomaterials and Cell Therapy, Sweden. Microscopy was performed at the Canadian Centre for Electron Microscopy at McMaster University, a facility supported by NSERC and other government agencies.

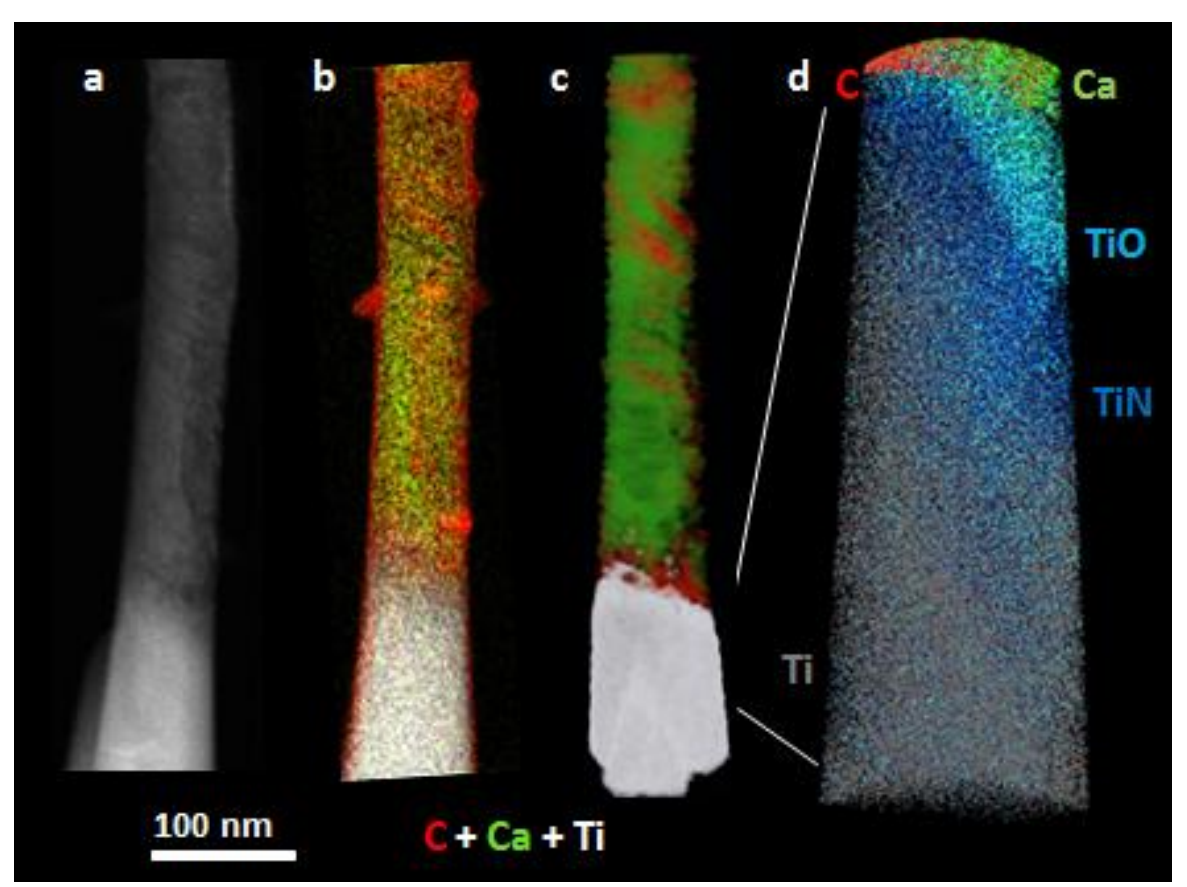

Figure 1. 4D EELS tomography and APT of human bone implant interface. (a) HAADF STEM image of needle-like sample. (b) A representative 2D EELS map and (c) corresponding 3D EELS tomogram, where red represents Carbon, green represents Calcium and white represents Titanium. (d) APT of a small fraction of the same sample. 